\title{
LEFT FREE WALL-SIDED MAHAIM TYPE FIBER AND AV NODAL REENTRY TACHYCARDIA - UNUSUAL BUT NOT IMPOSSIBLE COMBINATION
}

\author{
Milko Stoyanov $^{1}$ and Tchavdar Shalganov ${ }^{1}$ \\ ${ }^{1}$ Multiprofile Hospital for Active Treatment National Cardiology Hospital
}

January 5, 2022

\begin{abstract}
A 52-year-old patient with previous catheter ablation of AV nodal reentrant tachycardia (AVNRT) had a redo procedure for reported recurrence. During the study AVNRT was not inducible, but a previously unrecognized left-sided Mahaim type accessory pathway was diagnosed and ablated successfully.

\section{LEFT FREE WALL-SIDED MAHAIM TYPE FIBER AND AV NODAL REENTRY TACHY-} CARDIA - UNUSUAL BUT NOT IMPOSSIBLE COMBINATION

Milko Stoyanov, MD, Tchavdar Shalganov, MD, PhD

Cardiology Department, National Heart Hospital, 65 Konyovitsa Street, Sofia, Bulgaria

\section{Corresponding author:}

Dr. Milko Stoyanov, MD

Cardiology Department, National Heart Hospital

65 Konyovitsa St., 1309 - Sofia

E-mail: mil_ko@abv.bg

Key words:

Mahaim fiber; accessory pathway; AV nodal reentrant tachycardia; dual atrio-ventricular node physiology; left free wall; radiofrequency catheter ablation.

\section{Ethical approval:}

Written informed consent was obtained from the patient to publish this report in accordance with the journal's patient consent policy.

\section{Key clinical message}

Mahaim fibers have atrio-ventricular node like properties and are usually located around the tricuspid annulus. Rarely, they can be left-sided, around the mitral annulus. The combination of AV nodal reentry tachycardia with left-sided Mahaim fiber is uncommon and confusing combination which requires detailed electrophysiological knowledge for diagnosis and treatment.
\end{abstract}

\section{Abstract}


A 52-year-old patient with previous catheter ablation of AV nodal reentrant tachycardia (AVNRT) had a redo procedure for reported recurrence. During the study AVNRT was not inducible, but a previously unrecognized left-sided Mahaim type accessory pathway was diagnosed and ablated successfully.

\section{Introduction}

Mahaim fibers represent accessory pathways (APs) characterized by slow and exclusively antegrade decremental conduction, and lack of retrograde conduction. These APs comprise less than $3 \%-6 \%$ of all. ${ }^{1-3}$ Most Mahaim type APs are right sided with atrial insertion points at various sites along the tricuspid annulus. Left-sided Mahaim fibers have been occasionally reported with a prevalence of $1.6 \% .^{2}$ The preferred treatment is radiofrequency ablation. The most frequent coexisting arrhythmia is AV node reentrant tachycardia (AVNRT) which is a common finding in right sided Mahaim type APs, but has not been described in leftsided free wall fibers. ${ }^{2}$ We present a patient with a unique combination of AVNRT and left-sided Mahaim type AP.

\section{Clinical Case}

A 52-year-old Caucasian man with recurrent wide QRS complex tachycardia at 190 beats-per-minute was treated initially by catheter ablation for AVNRT six months ago (Figure 1). According to the discharge report dual AV node physiology was found and AVNRT was induced during the electrophysiological study. Modification of the slow pathway was done. During the procedure the coexistence of AP was not recognized. Medical history included a well-controlled arterial hypertension, duodenal ulcer, dyslipidemia on statin treatment, left anterior fascicular block (LAFB), incomplete right bundle branch block (RBBB), polypectomy for colon polyposis, hemorrhoidal disease and mild anemic syndrome. Coronary artery disease was excluded by prior coronary angiography. Physical examination, chest radiography and echocardiography revealed no underlying heart disease.

After a recurrence of the tachycardia a second electrophysiological study was carried out after informed written consent was obtained. At baseline, 12-lead electrocardiogram (ECG) showed sinus rhythm with incomplete RBBB and LAFB with no patent signs of ventricular preexcitation. PR and QRS intervals were $155 \mathrm{~ms}$ and $102 \mathrm{~ms}$, respectively. Standard multielectrode catheters were placed over the His bundle (HB), in the coronary sinus (CS) and right ventricular apex. During sinus rhythm the A-H and H-V intervals were $82 \mathrm{~ms}$ and $50 \mathrm{~ms}$, respectively.

Incremental and programmed ventricular pacing showed concentric and decremental conduction over the AV node with retrograde HB potential recorded (Figure 2A ). In sinus rhythm local preexcitation was noticed at the distal electrode pairs of the CS catheter (laterally-posterolaterally) where the local V potential preceded the $\mathrm{V}$ potential at the HB area and right ventricular apex (Figure 2A ).

Incremental atrial pacing performed from the proximal part of CS showed AV conduction 1:1 with increasing widening of the QRS complex, initial shortening followed by negativity of the $\mathrm{H}-\mathrm{V}$ interval, and simultaneous prolongation of the A-H and A-V intervals, respectively, consistent with slowly conducting AP with decremental properties (Figure 2B ).

Programmed CS stimulation with introduction of single and double premature extrastimuli induced a sudden prolongation of the A-H interval by $65 \mathrm{msec}$ with one echo-beat. No sustained tachycardias were induced at baseline and after pharmacological stimulation with hexoprenaline sulphate (only short bouts of wide QRS tachycardia with RBBB morphology was induced). A transseptal approach was used to map the location of the AP during sinus rhythm with a quadripolar 7F 4-mm tipped irrigated ablation catheter (Therapy CoolFlex L1, St. Jude Medical, USA). The mitral annulus was mapped and shortest local A-V interval in sinus rhythm was identified on the left free wall at 4 o'clock. Radiofrequency current (50 W for 120 $\mathrm{s}$, target temperature $42{ }^{\circ} \mathrm{C}$ ) was delivered at the target region inducing antegrade AP conduction block with disappearance of the local preexcitation (Figure 3). Following the ablation there was no recurrence of conduction over the AP and no tachycardia was induced by incremental and programmed atrial and ventricular stimulation before and after intravenous infusion of hexoprenaline sulphate. After administration 
of $24 \mathrm{mg}$ intravenous adenosine a transient complete AV block was induced.

The patient was discharged off antiarrhythmic drugs and has done well without any recurrences of tachycardia during a follow up of 10 months.

\section{Discussion}

Historically, in 1938, Mahaim and Benatt first described a special form of AP with unique electrophysiologic characteristics distinguishing them from other APs. ${ }^{4}$ Actually, Ivan Mahaim described only nodoventricular fibers. Other fibers like nodofascicular and fasciculoventricular were subsequently defined later. In 1988 Tchou et al. described the so-called atriofascicular $\mathrm{AP}^{5}$, which some authors called pseudo-Mahaim fibers. ${ }^{1}$ Most of them are with right-sided location while only sporadic cases of Mahaim fibers with left-sided location have been reported. Rare cases have been seen along the mitral annulus, mitral annulus-aorta junction and left coronary cusp. ${ }^{6-13}$

Below are listed the specific electrophysiological properties of Mahaim-like AP. No or minimal preexcitation is usually seen on ECG, there is no retrograde conduction over the AP, rapid atrial pacing elicits prolongation of the A-H, A-V and QRS intervals with shortening of the $\mathrm{H}-\mathrm{V}$ interval, antidromic A-V reentry tachycardia with various morphology and axis may be observed, and other heart conditions, especially dual A-V node pathway, may be associated. ${ }^{5}$ In such cases, the presence of AVNRT may complicate the appropriate diagnosis of the clinical tachycardia. The combination of dual A-V node physiology and left-sided Mahaim type fiber is not usual and has not been reported previously. In our patient we proved the persistence of dual A-V node physiology after the first ablation, but AVNRT was not inducible even after the administration of hexoprenaline.

The A-V node-like conduction properties of these APs are explained by the embryological development process of the $\mathrm{A}-\mathrm{V}$ node and the $\mathrm{A}-\mathrm{V}$ conduction axis. They come from the "primary ring" and contribute only to the tricuspid annulus. Mahaim fibers originate from the embryotic remnants of this "primary ring". These explain the slow conduction properties and most frequent right-sided location. The left-sided location of Mahaim APs may be due to leftward extension of the A-V node or to a defect in embryological migration accompanied by an A-V isolation defect, as it is with left-sided AVNRTs. ${ }^{2}$

We describe an unusual, previously unreported, left free wall-sided AP conducting only antegradely with slow decremental properties, in combination with dual A-V nodal pathway and previously ablated AVNRT. The AP was successfully and uncomplicatedly ablated on the left free wall of the mitral ring. This patient was previously not fully diagnosed during electrophysiological study when non-sustained wide QRS complex tachycardia was induced. We were able to find two cases in which Mahaim-associated wide QRS tachycardias were misdiagnosed as ventricular tachycardia. ${ }^{7,8}$ Incremental atrial pacing is essential for identifying the presence of ventricular preexcitation not only in patients with wide complex tachycardia. Rapid atrial pacing rate is expected to unmask or increase the degree of preexcitation via delayed conduction through the A-V node. Simultaneously, the progressive lengthening of A-H and A-V intervals, respectively, is characteristic for Mahaim-like AP and crucial for the correct diagnosis.

\section{Conclusion}

Mahaim-type APs are uncommon. Usually, they are right-sided, but rarely can be left-sided. The combination of left free wall-sided Mahaim-type AP, dual A-V node physiology and AVNRT has not been described previously. Detailed electrophysiological knowledge is needed for proper diagnosis, and for successful and uncomplicated treatment with curative radiofrequency catheter ablation.

Disclosures: The authors do not report any conflict of interest regarding this work.

Funding sources: The authors have no funding sources to disclose.

Author Contribution: Prof. Tchavdar Shalganov: involved in conception, and design, interpretation of data and critical revision of the article. 
Milko Stoyanov: involved in writing the article and interpretation of data.

\section{ORCID:}

Milko Stoyanov https://orcid.org/0000-0002-1226-6119

Tchavdar Shalganov https://orcid.org/0000-0003-2268-0759

\section{References}

1. Szumowski L, Bodalski R, Jedynak Z, et al. 2008. The clinical course and risk in patients with pseudoMahaim fibers. Cardiol J 15:365-370.

2. Ozcan EE, Turan OE, Akdemir B, et al. 2021. Comparison of electrophysiological characteristics of right and left-sided Mahaim-type accessory pathways. J Cardiovasc Electrophysiol 32:360-369.

3. Lee KN, Kim YH. 2017. Preexcitation syndrome with a Mahaim-type accessory pathway. Int J Arrhythm 18:151-154.

4. Balaji S, Tchou P, Kanter R. 2020. Mahaim fibers: should they be renamed? Heart Rhythm 17:161-162.

5. Tchou P, Lehmann MH, Jazayeri M, Akhtar M. 1988. Atriofascicular connection or a nodoventricular Mahaim fiber? Electrophysiologic elucidation of the pathway and associated reentrant circuit. Circulation $77: 837-848$.

6. Francia P, Pittalis MS, Ali H, Cappato R. 2008. Electrophysiological study and catheter ablation of a Mahaim fibre located at the mitral annulus-aorta junction. J Interv Card Electrophysiol 23:153-157.

7. Osman F, Stafford PJ, Ng GA. 2009. Looks like VT but isn't - successful ablation of a left free wall accessory pathway with Mahaim-like properties. Indian Pacing Electrophysiol J 9:112-118.

8. Wilsmore BR, Tchou PJ, Kanj M, Varma N, Chung MK. 2012. Catheter ablation of an unusual decremental accessory pathway in the left coronary cusp of the aortic valve mimicking outflow tract ventricular tachycardia. Circ Arrhythm Electrophysiol 5:e104-e108.

9. Galeazzi M, Russo M, Ricili S, Lavalle C, Pandozi C. 2012. Electrophysiologic behavior of a left sided accessory pathway with decremental (Mahaim-like) properties. Open Journals of Internal Medicine $2: 34-36$.

10. Hluchy J, Schikel S, Jorger U, Jurkovicova O, Sabin GV. 2000. Electrophysiologic characteristics and radiofrequency ablation of concealed nodofascicular and left antegrade atriofacicular pathways. J Cardiovasc Electrophysiol 11:211-217.

11. Tada H, Nogami A, Naito S, Oshima SH, Taniguchi K, Kutsumi Y. 1999. Left posteroseptal Mahaim fiber associated with marked longitudinal dissociation. Pacing Clin Electrophysiol 22:1696-1699.

12. Jonson CT, Brooks C, Jaramillo J, Mickelsen S, Kusimoto FM. 1997. A left free-wall, decrementally conducting, atrioventricular (Mahaim) fiber: diagnosis at electrophysiological study and radiofrequency catheter ablation guided by direct recording of a Mahaim potential. Pacing Clin Electrophysiol 20[Pt. I]:2486-2488.

13. Goldberger JJ, Pederson DN, Damle RS, Kim YH, Kadish AH. 1994. Antidromic tachycardia utilizing decremental, latent accessory atrioventricular fibers: differentiation from adenosine-sensitive ventricular tachycardia. J Am Coll Cardiol 24(3);732-8.

\section{Legend to the figures:}

Figure 1. Outpatient ECG during tachycardia before the first ablation. On the left panel - peripheral leads, on the right panel - precordial leads. Paper speed $25 \mathrm{~mm} / \mathrm{sec}$.

Figure 2. Panel A - Programmed ventricular stimulation during the second electrophysiological study demonstrates concentric and decremental retrograde conduction with retrograde His bundle potential. The sinus beat shows local preexcitation at the distal CS. Shown are ECG leads I, aVF and V1, and intracardiac electrograms from the His bundle ( $\mathrm{His}_{1-2}$, His $2-3$, His $\left.3-4\right)$, coronary sinus $\left(\mathrm{CS}_{1-2}, \mathrm{CS}_{3-4}, \mathrm{CS}_{5-6}\right.$. $\left.\mathrm{CS}_{7-8} . \mathrm{CS}_{9-10}\right)$ and right ventricular apex $\left(\mathrm{RVA}_{1-2}, \mathrm{RVA}_{3-4}\right)$. Panel $\mathbf{B}$-Incremental stimulation from the proximal part of the coronary sinus $\left(\mathrm{CS}_{9-10}\right)$ with cycle length $320 \mathrm{~ms}$. An increasing widening of QRS complex with shortening of $\mathrm{H}-\mathrm{V}$ interval and simultaneous prolongation of the $\mathrm{A}-\mathrm{H}$ and $\mathrm{A}-\mathrm{V}$ intervals are seen. The $\mathrm{A}-\mathrm{V}$ interval of 
$2^{\mathrm{d}}, 3^{\text {th }}$ and $4^{\text {th }}$ paced complex is $150 \mathrm{~ms}, 165 \mathrm{~ms}$ and $187 \mathrm{~ms}$, respectively. The corresponding $\mathrm{H}-\mathrm{V}$ intervals of the same complexes are $15 \mathrm{~ms}, 5 \mathrm{~ms}$ and $-7 \mathrm{~ms}$. The $\mathrm{V}$ potential in RVA is later than the $\mathrm{V}$ potential in the His bundle area. Small Mahaim potential (M) can be seen at $\mathrm{CS}_{3-4}$. Shown are the same ECG leads and intracardiac electrocardiograms as in Figure 2A.

Figure 3. Loss of local preexcitation during radiofrequency application. The CS catheter is at a deeper position. Shown are the same ECG leads and intracardiac electrocardiogram as in Figure 2A.
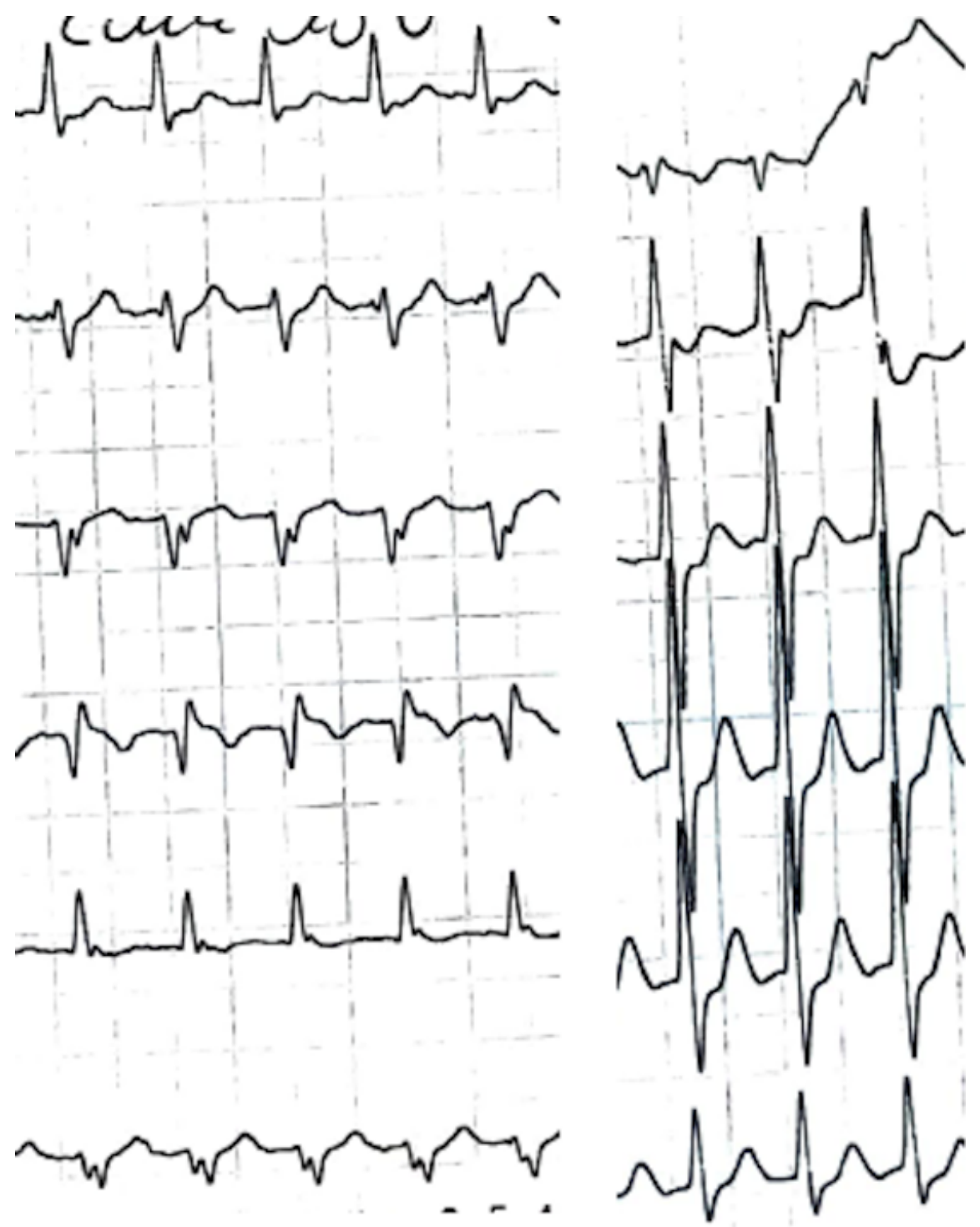


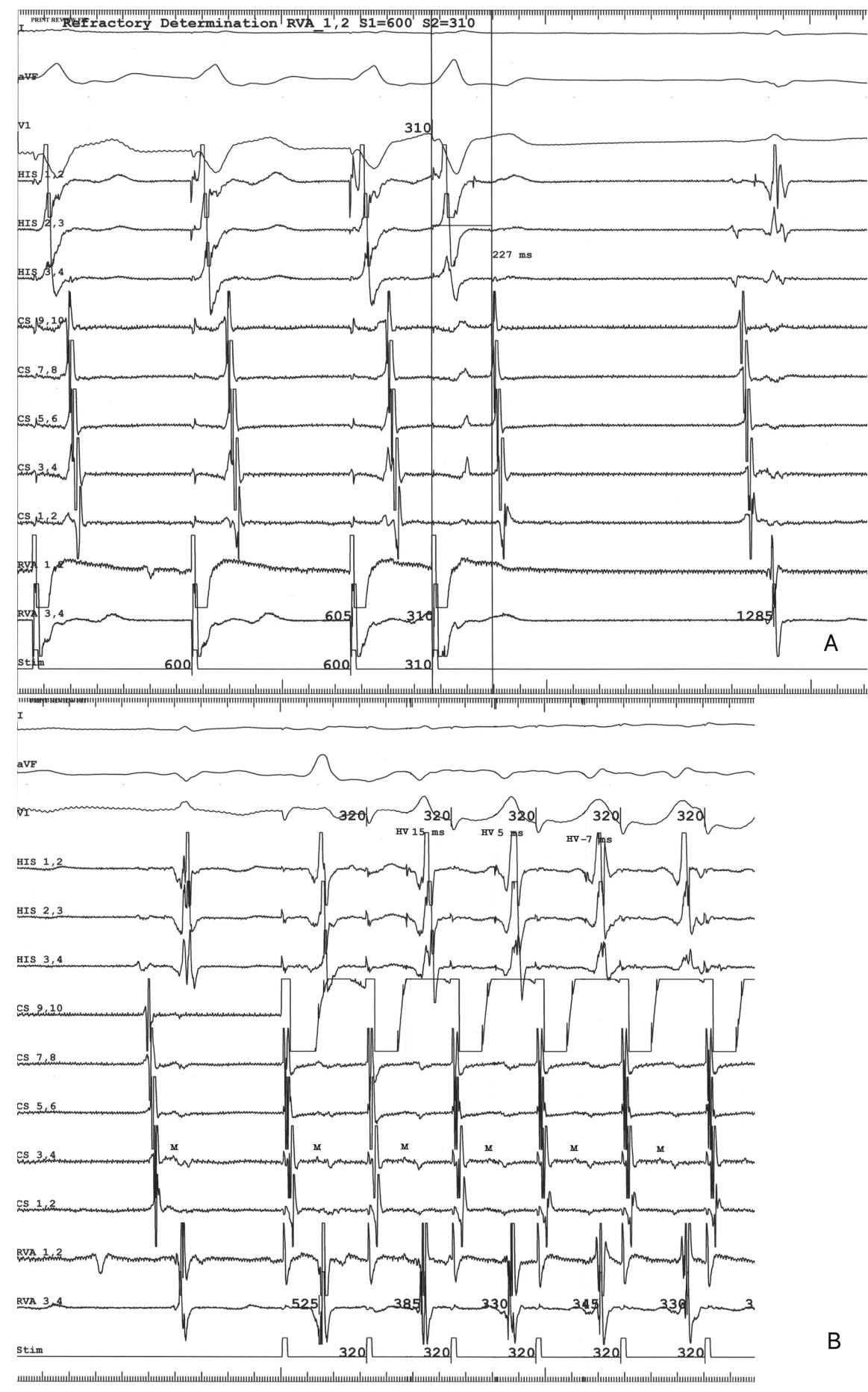




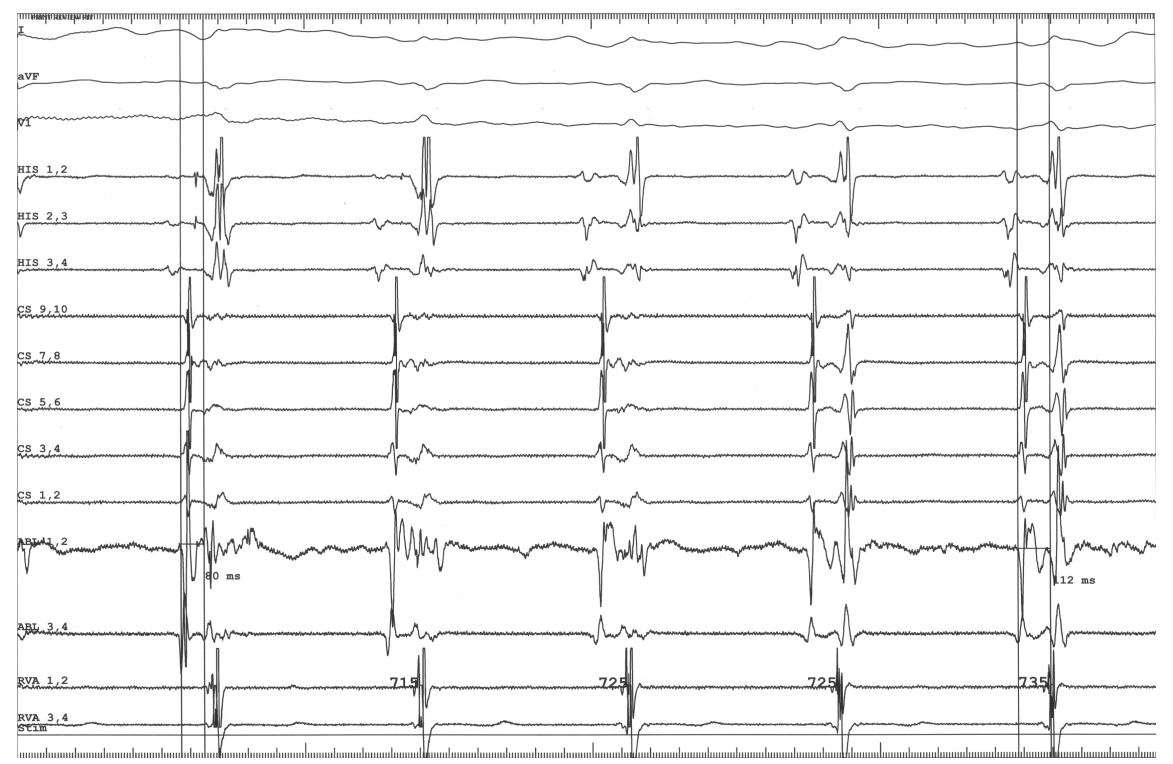

